\title{
Imitation Mathematical Model of Construction \\ of Two-Dimensional Radar-Image Images \\ of Air Objects in Interest of Assessment \\ of Quality of Recognition
}

\author{
Valerii P. Berdyshev"a , Aleksandr M. Mironov, \\ Oleg N. Pomazuev ${ }^{\mathrm{b}}$, Aleksei N. Saveliev', \\ Vladimir A. Kopylov ${ }^{\mathrm{d}}$ and Vitalii V. Loy ${ }^{\mathrm{d}}$ \\ ${ }^{a}$ Military Academy of Aero-Space Defense \\ named after the Marshal of Soviet Union G.K. Zhukov \\ 50 Zhigareva Str., Tver, 170022, Russia \\ ${ }^{b}$ Main Department of Scientific and Research Activities \\ and Technological Support of the Advanced Technologies \\ of the Ministry of Defense of the Russian Federation \\ 84/32 Profsojznaj Str., Moscow, 117997, Russia \\ "JSC "Airborne navigation systems" \\ 15, 12 Bolshaya Novodmitrovskaya Str., Moscow, 127015, Russia \\ ${ }^{d}$ Siberian Federal University \\ 79 Svobodny, Krasnoyarsk, 660041, Russia
}

Received 29.01.2018, received in revised form 25.08.2018, accepted 12.10.2018

Currently, the task of target recognition is of increasing interest, especially among developers of air traffic control and air defense systems. The article is devoted to the development of an imitative mathematical model for the construction of two-dimensional radar images of airborne objects in the interest of assessing the quality of recognition in survey radars. The proposed simulation model for constructing two-dimensional radar images of airborne objects makes it possible to carry out comprehensive studies of portraits of 19 types of airborne objects of foreign and Russian production with a change in the angles of location, the nature and parameters of the flight instabilities of flight, the signal-to-noise ratio, the parameters of the radar probing signals, and range to the target. It is shown that in the absence of destabilizing factors (uneven rotation, radial displacement, presence of noise and noise, etc.), the two-dimensional radar image of air objects being formed adequately reflects the structure of the target. The obtained results can be used in developing and assessing the quality of systems for recognizing classes and types of air assets based on signaling characteristics in existing and prospective radars.

Keywords: algorithm, face, portrait, portrait of dalnostnyj radar, azimuthal portrait, twodimensional radar imageapplication, of inverse aperture synthesis, multi-frequency probe signal, detection rate.

(C) Siberian Federal University. All rights reserved

This work is licensed under a Creative Commons Attribution-NonCommercial 4.0 International License (CC BY-NC 4.0).

* Corresponding author E-mail address: berd696969@mail.ru 
Citation: Berdyshev V.P., Mironov A.M., Pomazuev O.N., Saveliev A.N., Kopylov V.A., Loi V.V. Imitation mathematical model of construction of two-dimensional radar-image images of air objects in interest of assessment of quality of recognition, J. Sib. Fed. Univ. Eng. technol., 2018, 11(7), 764-774. DOI: 10.17516/1999-494X-0090.

\title{
Имитационная математическая модель построения двумерных радиолокационных изображений воздушных объектов в интересах оценки качества распознавания
}

\author{
В.П. Бердышев ${ }^{a}$, А.М. Миронов ${ }^{\sigma}$, О.Н. Помазуев ${ }^{\sigma}$,
} А.Н. Савельев ${ }^{\text {, }, \text { В.А. Копылов }}$, В.В. Лойг

${ }^{a}$ Военная академия воздушно-космической обороны им. Маршала Советского Союза Г.К. Жукова Россия, 170022, Тверь, ул. Жигарева, 50

${ }^{\sigma}$ Главное управление научно-исследовательской деятельности и технологического сопровождения передовых технологий МО РФ Россия, 117997, Москва, ул. Профсоюзная, 84/32 ${ }^{8} \mathrm{OAO}$ «Бортовые аэронавигачионные системы»» Россия, 127015, Москва, ул. Большая Новодмитровская, 12, стр. 15 ${ }^{2}$ Сибирский федеральный университет Россия, 660041, Красноярск, пр. Свободный, 79

В настоящее время задача распознавания иелей вызывает повышенный интерес, особенно у разработчиков систем управления воздушным движением и противовоздушной обороны. Статья посвящена разработке имитационной математической модели построения двумерных радиолокационных изображений воздушных объектов в интересах оценки качества распознавания в обзорных РЛС. Предложенная имитационная математическая модель построения двумерных радиолокационных изображений воздушных объектов позволяет проводить всесторонние исследования портретов 19 типов воздушных объектов зарубежного и российского производства при изменении ракурсов локации, характера и параметров траекторных нестабильностей полета, отношения сигнал/шум, параметров зондирующих сигналов РЛС с учетом ошибок измерения радиальной скорости и дальности до цели. Показано, что вусловиях отсутствия дестабилизирующих факторов (неравномерности поворота, радиального перемещения, наличия шумов и помех и т. п.) формируемое двумерное радиолокационное изображение воздушных объектов адекватно отражает структуру цели. Полученные результаты могут быть использованы при разработке и оценке качества систем распознавания классов и типов воздушных объектов по сигнальным признакам в существующих и перспективных РЛС.

Ключевыеслова: алгоритм, распознавание, радиолокационныйпортрет, дальностныйпортрет, азимутальный портрет, двумерное радиолокационное изображение, инверсноесинтезирование апертуры, многочастотный зондирующий сигнал, вероятность распознавания.

Разработка систем распознавания, в частности радиолокационного распознавания, сопряжена с решением целого ряда задач. Центральным является вопрос о словаре признаков, на 
языке которых производятся как априорные описания классов ВО, так и апостериорное описание распознаваемых объектов. Связано это с тем, что рабочий словарь признаков определяет состав и технические характеристики измерительных средств системы распознавания, в данном случае РЛС, которые считаются наиболее ресурсоемкими элементами системы. Решение задачи распознавания и идентификация целей по их изображениям, в том числе и по радиолокационным, зависит от возможности выделения на изображении характерных признаков объекта наблюдения. Такими признаками объектов служат пространственные признаки (размер и форма объекта), а также яркостные признаки. Для увеличения числа распознаваемых классов целей и повышения достоверности получаемой информации необходимо увеличивать количество информативных признаков и соответствующие вычислительные ресурсы на их обработку $[1-4,8,9]$.

В настоящее время значительное распространение получили цифровые методы моделирования вторичного излучения реальных воздушных целей с использованием компьютерной техники. Однако результаты, полученные при имитационном моделировании, должны уточняться в ходе натурных экспериментов. Как показывают исследования, степень совпадения результатов моделирования и натурных экспериментов при узкополосном зондировании для ЭПР составляет 0-2,5 дБ, для угловых и частотных корреляционных функций с точностью до 10-30 \% (в зависимости от типа цели). Например, коэффициенты корреляции ДП при ЛЧМзондировании, полученные при моделировании и экспериментально, составили 0,88-0,97. Полученные результаты подтверждают целесообразность применения имитационного математического моделирования для исследований отражений радиоволн от ВО $[4,5]$. В связи с этим в настоящей работе распознавание классов (типов) ВО предлагается проводить статистическими методами с использованием признаков, извлеченных из ДРЛИ. Указанные признаки должны отвечать следующим требованиям: наиболее полно характеризовать как геометрические, так и отражательные особенности ВО; не вызывать сложностей при определении их численных значений; обеспечивать вероятность распознавания целей в рамках принятого алфавита классов не ниже заданной.

Наиболее исследованными и отвечающими указанным требованиям признаками распознавания по ДРЛИ являются:

- количество разрешаемых рассеивающих центров (РЦ) в изображении;

- плотность расположения РЦ; максимальное расстояние между наиболее удаленными РЦ;

- сумма расстояний между всеми РЦ;

- усредненное расстояние между РЦ;

- площадь ВЦ, аппроксимированная кругом.

Результаты распознавания (оценки вероятностей) при использовании этих признаков достаточно высоки, однако они получены при следующих ограничениях [6, 7]:

- значения курсовых углов полета целей выбирались не менее $30^{\circ}$;

- отсутствовал учет возможных затенений РЦ крупными элементами конструкции планера;

- отсутствовал учет искажений, связанных с проявлением ТН полета, или возникающих ошибок их компенсации при угловых скоростях рысканий более $0,5 \%$;

$$
-766-
$$


- отсутствовал учет ошибок измерения радиальной скорости, дальности до цели и др.

При решении задач распознавания целей по их ДРЛИ для получения адекватных оценок перечисленные выше факторы и условия должны быть обязательно учтены.

Порядок получения и анализа характеристик радиолокационных портретов (РЛП) ВО (дальностного, азимутального, двумерного и трехмерного) приведен в многочисленных публикациях, относящихся к теории распознавания, например [1-4, 6, 7].

Цель статьи - предложить имитационную математическую модель построения двумерных радиолокационных изображений воздушных объектов в интересах оценки качества распознавания.

В соответствии с целевым назначением к имитационной математической модели (ИММ) предъявлялись следующие требования: возможность проведения исследований при любых ракурсах локации; возможность имитации движения цели по различным траекториям (высота, скорость, дальность) при наличии помех и внутренних шумов приемника различного уровня.

Рассмотрим назначение каждого блока ИММ и содержание проводимых в них операций (рис. 1).

Блок 1 (заставка), вид которой показан на рис. $2 a$.

Блок 2 предназначен для выбора типа цели, задания начальных параметров движения цели, характеристик РЛС, траектории полета цели, траекторных нестабильностей, помех и внутренних шумов приемника. В этот блок вводятся следующие начальные данные (рис. 2б): тип цели; значения параметров траектории движения цели для РЛС (курс цели, наклонная дальность до цели, высота цели, скорость цели, амплитуда и угловая скорость рысканий); значения параметров РЛС: несущая частота, количество частот многочастотного зондирующего сигнала (МЧЗС), диапазон перестройки частоты, период повторения импульсов, время инверсного радиолокационного синтезирования апертуры (ИРСА), амплитуда внутренних шумов приемника. Исходные и рассчитанные данные из этого блока поступают в блок 3.

В блоке 3 (моделирование траектории движения цели) моделируется полет ВО в турбулентной атмосфере, т.е. рассчитываются координаты ВО относительно РЛС и углы, определяющие положение ВО в пространстве. Полет ВО имитируется в связанной с РЛС прямоугольной системе координат 0XYZ с осями 0X и 0Z, расположенными в горизонтальной плоскости (рис. 3).

Начало отсчета системы координат $O X Y Z$ совпадает с точкой стояния РЛС. Геометрические и отражательные особенности цели определяются в подвижной системе координат охуz, связанной с ЦСЦ ВО. Ось ох направлена вдоль фюзеляжа в сторону, противоположную линейной скорости цели, ось $o z$ - по размаху крыла перпендикулярна оси $o x$, а ось $о y$ составляет с остальными осями правую ортогональную систему координат. Ее ориентация и положение в пространстве относительно системы координат $0 X Y Z$ определяются координатами $\mathrm{X}_{0}, \mathrm{Y}_{0}, \mathrm{Z}_{0}$, углами рыскания - $\psi$, тангажа $-\vartheta$ и крена $-\xi$. Угол $\psi$ определяет поворот ВО в плоскости X0Z, угол $\vartheta-$ в плоскости $X O Y$, угол $\xi-$ в плоскости $Z O Y$.

При полете ВО в турбулентной атмосфере возникают ТН. Определяющее влияние на флюктуации ЭПР оказывают рыскания ВО (табл. 1). Максимальные спектральные плотности угловой скорости рысканий составляют величину порядка 4-4,5\%. 


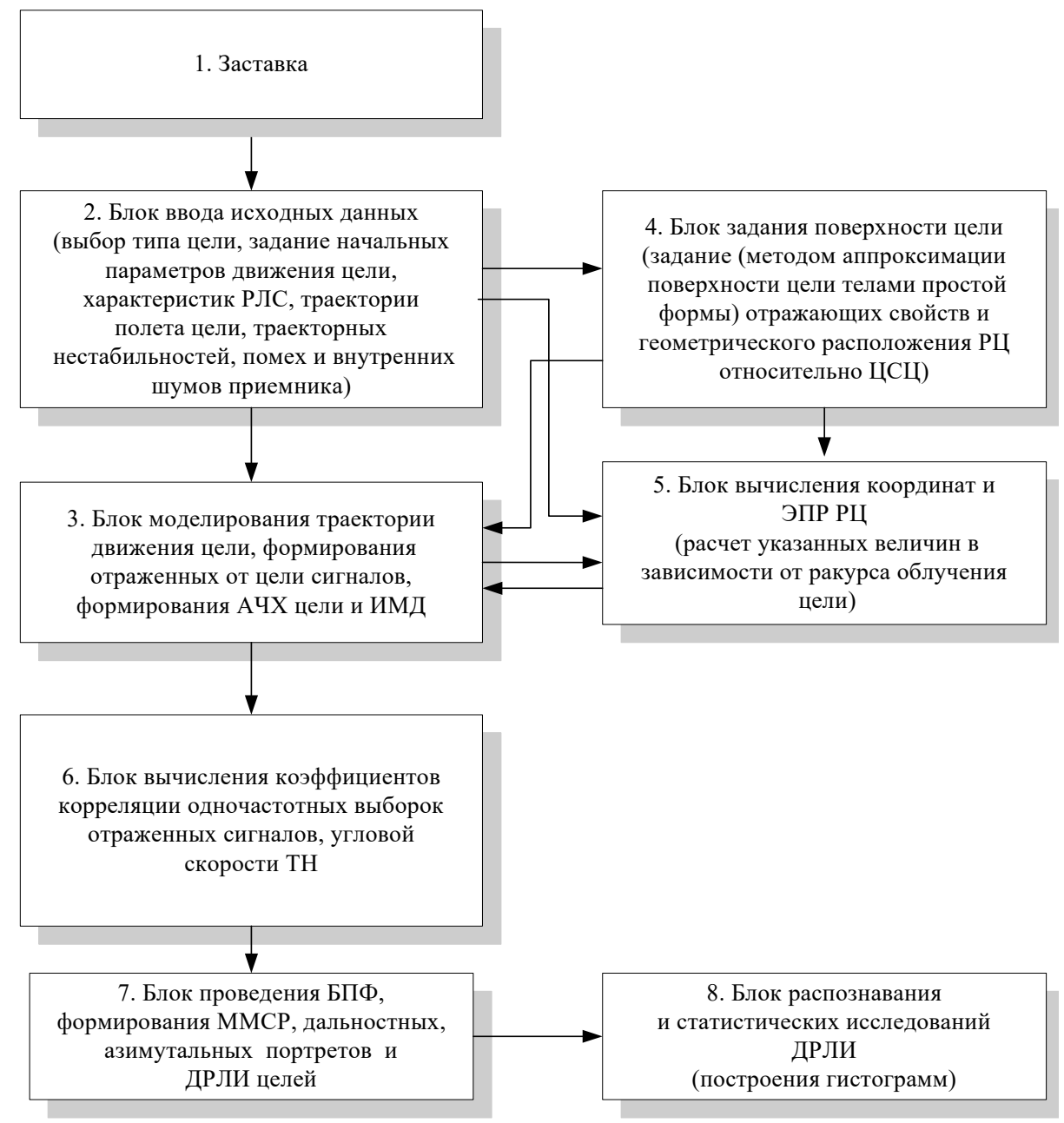

Рис. 1. Структурная схема имитационной математической модели

Fig. 1. Block diagram simulation mathematical model

Таблица 1. Влияние на флюктуации эффективной поверхности рассеяния рысканий воздушных объектов

Table 1. The effect on the fluctuations of the effective scattering surface of airborne objects

\begin{tabular}{|c|c|c|c|}
\hline \multirow{2}{*}{ Режим управления самолетом } & \multicolumn{3}{|c|}{ Аэродинамическая характеристика, град } \\
\cline { 2 - 4 } & $\begin{array}{c}\sigma_{\vartheta} \\
\text { (тангаж) }\end{array}$ & $\begin{array}{c}\sigma_{\xi} \\
\text { (крен) }\end{array}$ & $\begin{array}{c}\sigma_{\psi} \\
\text { (рыскания) }\end{array}$ \\
\hline Летчик & 0,82 & 3,43 & 3,52 \\
\hline Автопилот с корректором высоты & 0,99 & 0,67 & 0,37 \\
\hline Автопилот без корректора высоты & 1,28 & 0,85 & 0,53 \\
\hline
\end{tabular}

* $\sigma$ - среднеквадратическое отклонение величины угла. 


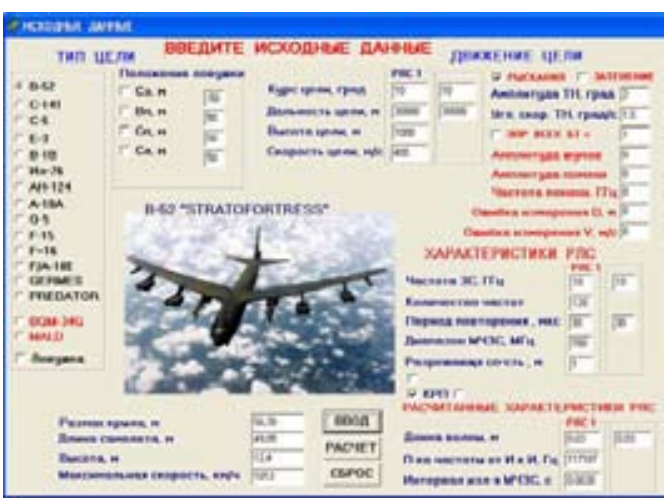

a)

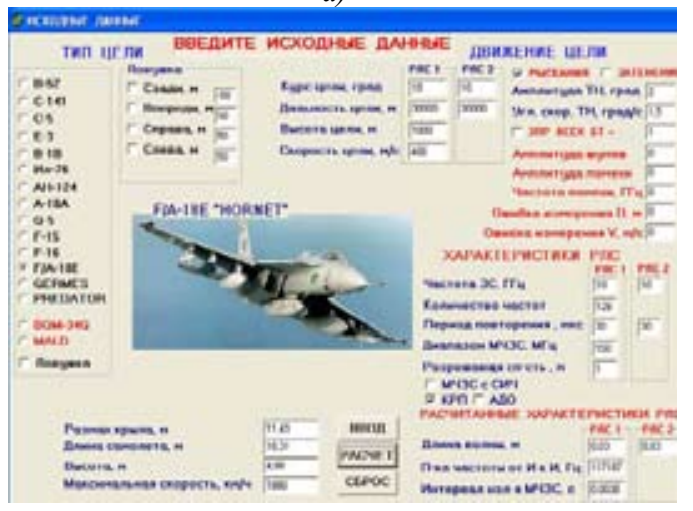

в)

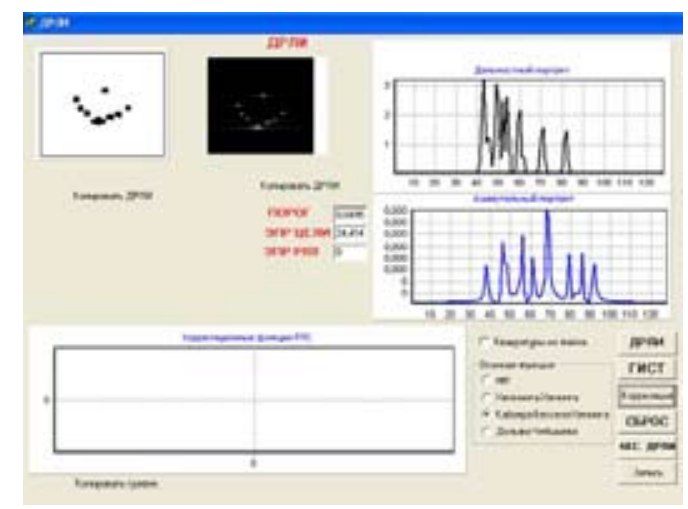

б)

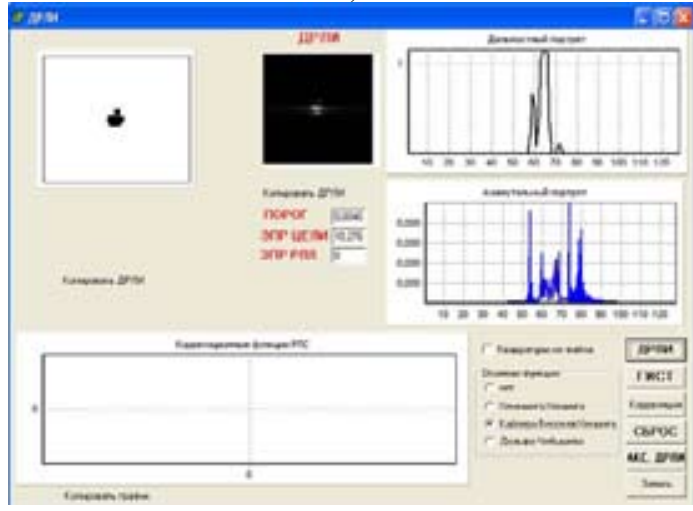

2)

Рис. 2. Вид интерфейса ввода исходных данных заставки (а), (в) и радиолокационных портретов воздушных объектов В-52 b F/A-18E (б), (г)

Fig. 2. View of the interface for input of the initial data of the screen saver (a), (B) and radar portraits of air objects B-52 b F/A-18E (б), (г)

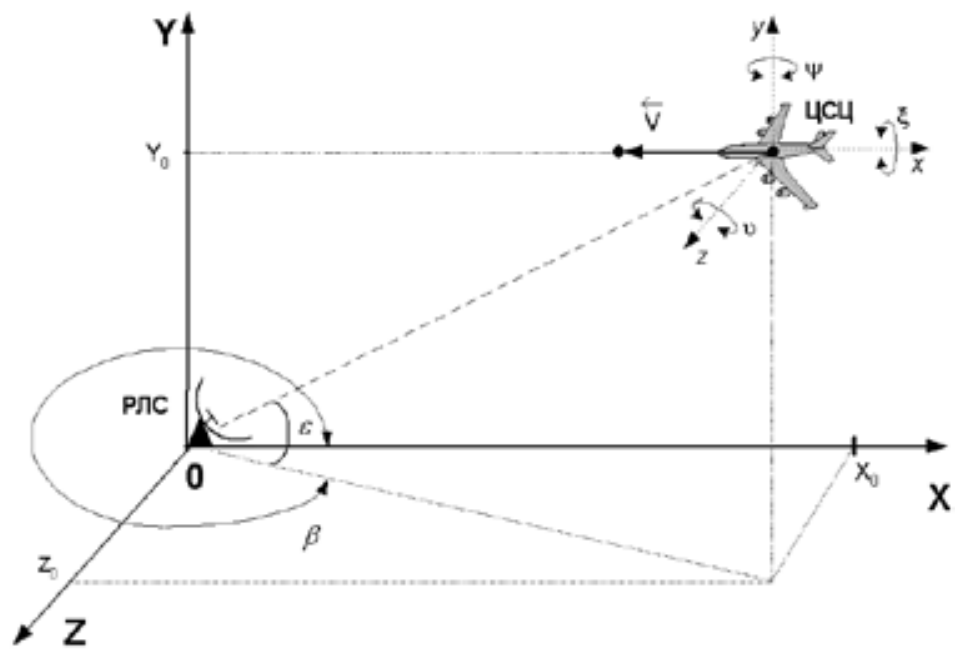

Рис. 3. Система координат при моделировании движения цели

Fig. 3. Coordinate system modeling traffic goals 
Для задания траектории ВО выберем следующие значения параметров движения:

$\psi=\mathrm{A} \cdot \sin \left(\gamma \cdot \mathrm{t}+\varphi_{0}\right), \vartheta=0, \xi=0, \mathrm{~V}=\mathrm{V}_{0}=$ const, $\mathrm{X}=\mathrm{X}_{0} \pm \mathrm{Vt}, \mathrm{Y}=\mathrm{Y}_{0}, \mathrm{Z}=\mathrm{Z}_{0}$, где $\varphi_{0}$ - начальная фаза угла рысканий.

Таким образом, в блоке 3 рассчитываются координаты ВО относительно РЛС и углы, определяющие положение ВО в пространстве.

В блок 4 (задания поверхности цели, согласно [5]) из блока 2 (исходных данных) поступает информация о типе выбранной цели. В блоке 4 содержится описание поверхности цели совокупностью расположенных определенным образом в пространстве тел простой формы. Информация представляет собой перечень элементов, составляющих поверхность ВО: геометрические размеры тел простой формы; исходные значения для расчета их ЭПР; начальные координаты, определяющие положение РЦ на поверхности ВО, углы видимости РЦ и координаты вершин экранов затенения.

В блок 5 (вычисления координат и ЭПР РЦ) из блока 2 поступают заданные характеристики РЛС. Из блока 3 - координаты ВО относительно РЛС и углы, определяющие положение ВО в пространстве. Из блока 4 - описание совокупности расположенных определенным образом в пространстве тел простой формы. На основе поступивших данных методом аппроксимации поверхности цели телами простой формы проводится расчет координат и ЭПР каждого РЦ на основе выражений, зависящих от вида аппроксимирующего тела. Затем ЭПР РЦ (тел простой формы) и их координаты пересчитываются в систему координат РЛС с учетом траектории полета и углового положения цели относительно РЛС. Рассчитанные в соответствии с ракурсом координаты и ЭПР РЦ поступают в блок 3. В этом блоке рассчитываются значения амплитуд и фаз, отраженных от ВЦ сигналов путем векторного сложения отражений от РЦ. По рассчитанным значениям амплитуд и фаз формируется КЧХ цели, ИМД и одночастотные выборки отраженных сигналов.

В блок 6 (вычисления коэффициентов корреляции, оценки угловой скорости ТН) из блока 3 поступают иммитационная матрица данных (ИМД) и одночастотные выборки отраженных сигналов РЛС. С полученными данными проводится корреляционный анализ.

В блоке 7 (проведения БПФ, формирования ММСР, ДП, АП и ДРЛИ целей) формируется матрица многочастотно-синтезированного рассеяния (ММСР), проводится двумерное быстрое преобразование Фурье (БПФ). В результате формируются дальностные портреты (ДП) и азимутальные (АП), ДОР и ДРЛИ цели (рис. 22). На рис. 4 представлены ДРЛИ В-52, В-1В, F-18Е и Predator при ракурсе q $=10^{\circ}$ (а), $50^{\circ}$ (б) и $90^{\circ}$ (в) соответственно, а на рис. 5 - ДРЛИ В-52, С-141, С-5 и Е-3 при ракурсе $10^{\circ}$ (а) и $50^{\circ}$ (б).

В блок 8 (распознавания и статистических исследований ДРЛИ) из блока 7 поступают значения, выражающие собой ММСР, на основе которых производится распознавание цели и построение гистограмм распределения (рис. 6).

Изучение особенностей различных признаков позволило для проведения дальнейших исследований и получения оценок распознавания предложить следующую признаковую совокупность (рис. 4 и 5): площадь многоугольной фигуры, образованной ДРЛИ, $-\mathrm{S}$; количество разрешаемых РЦ на ДРЛИ - M; расстояние между наиболее удаленными РЦ - R; ЭПР как сумма ЭПР всех РЦ - $\sigma$.

ИММ позволяет проводить оценку качества ДРЛИ. На рис. 7 представлены ДРЛИ цели С-141 при ракурсе q=10 при отношении сигнал/шум: 4 дБ, 2 дБ, 0 дБ и -2дБ соответственно 

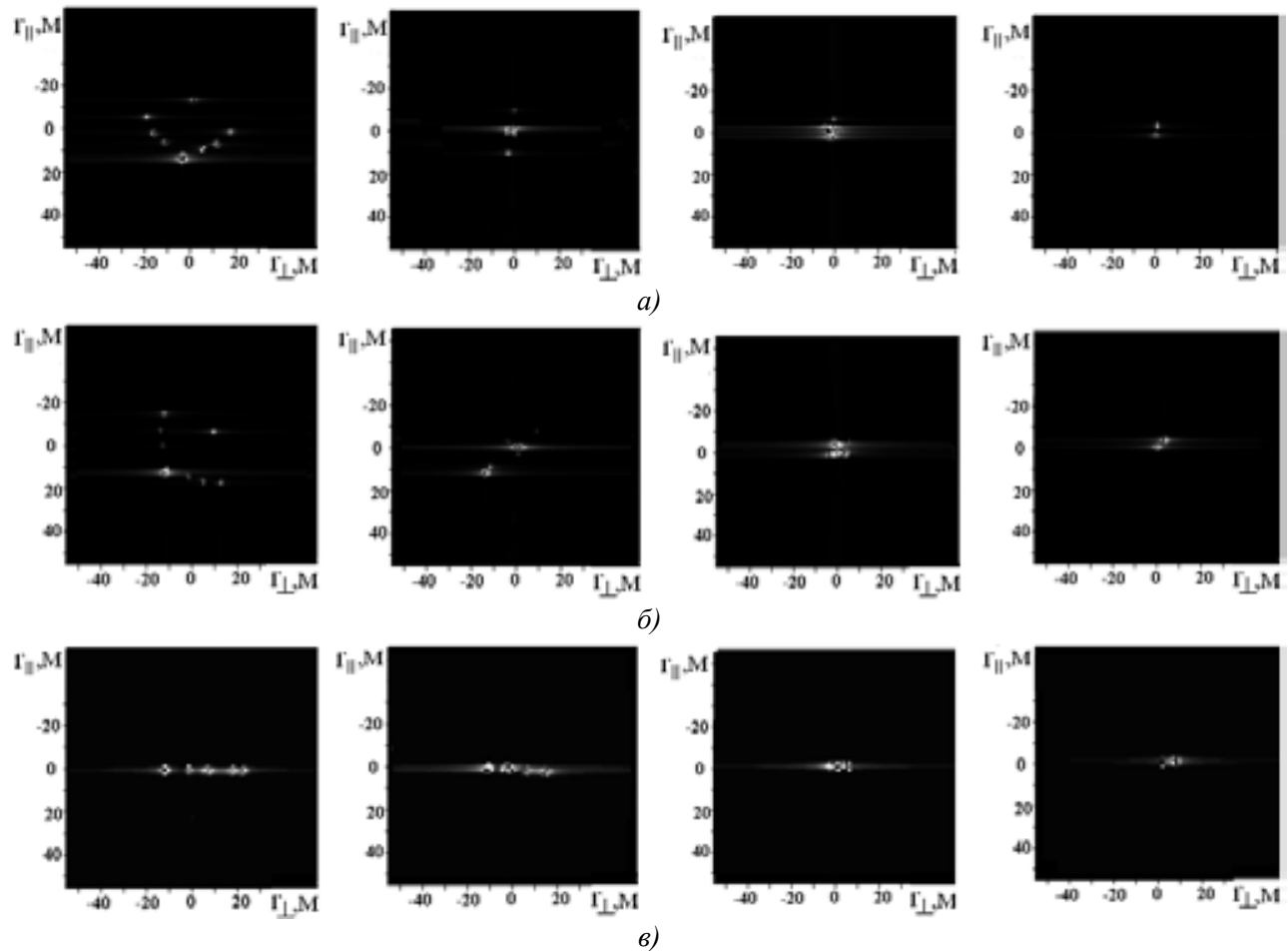

Рис. 4. Двумерные радиолокационные изображения B-52, B-1B, F-18E и Predator при различном ракурсе

Fig. 4. Two-dimensional radar images of the B-52, B-1B, F-18E and Predator at different perspective
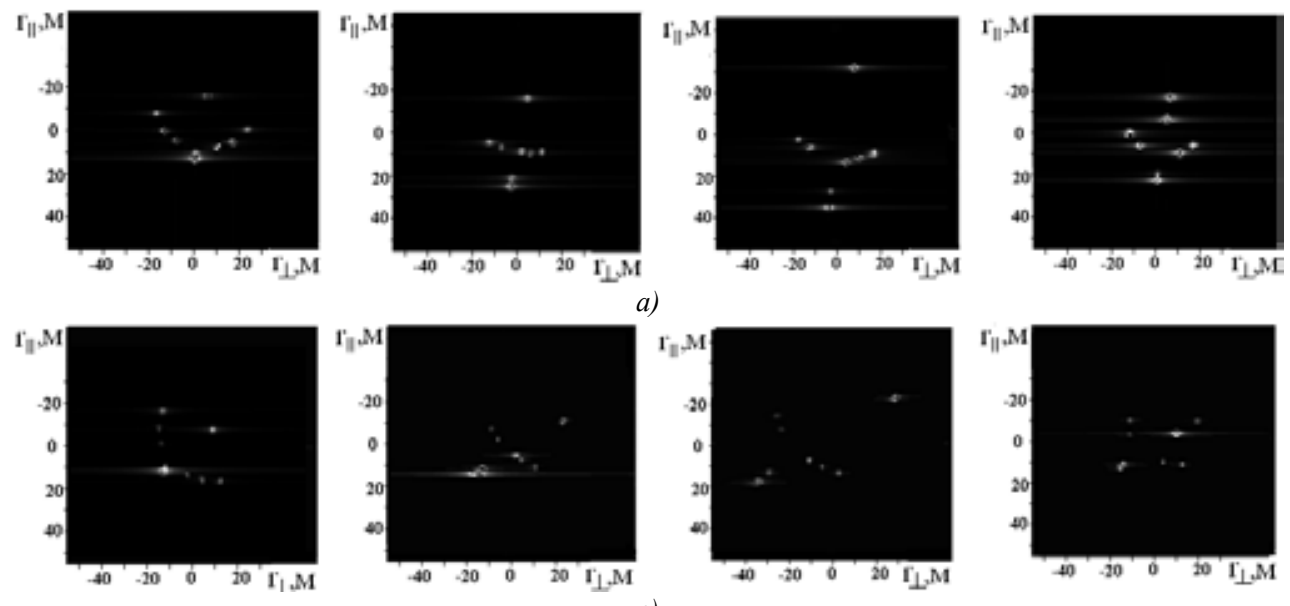

Рис. 5. Двумерные радиолокационные изображения В-52, C-141, C-5 и Е-3 при различном ракурсе

Fig. 5. Two-dimensional radar images of the B-52, C-141, C-5 and the E-3 with a different perspective 

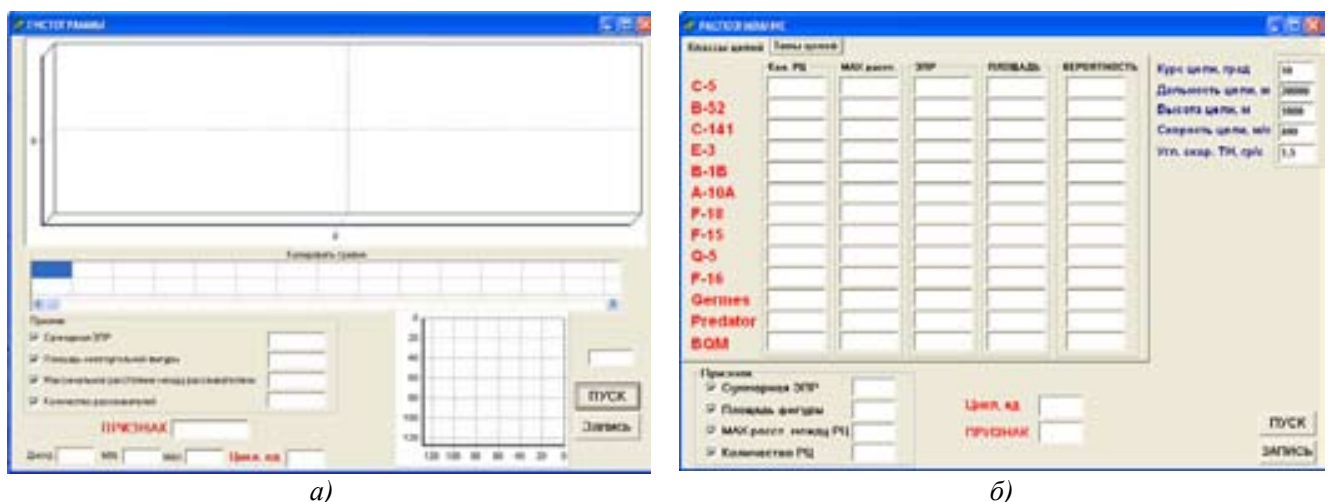

Рис. 6. Вид интерфейса построения гистограмм (а) и распознавания классов и типов воздушных объектов (б)

Fig. 6. View of the interface for constructing histograms (a) and recognizing the classes and types of air objects (б)
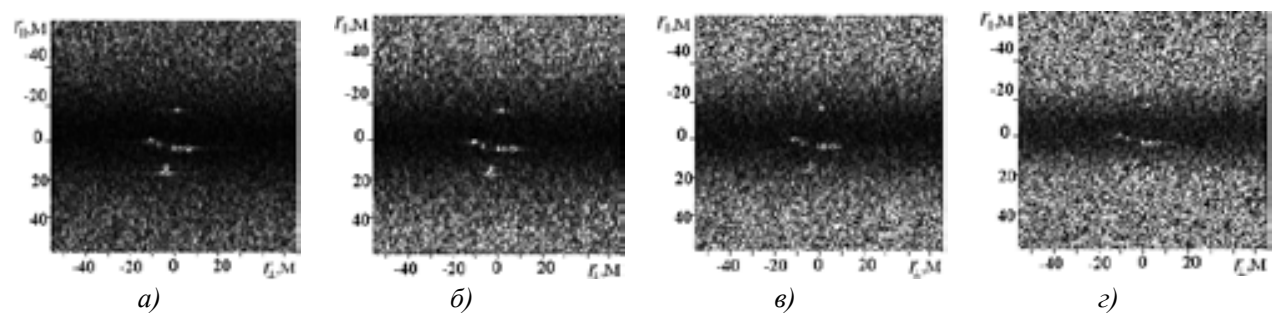

Рис. 7. Двумерные радиолокационные изображения цели С-141 при различном отношении сигнал/шум

Fig. 7. Two-dimensional radar images of the target C-141 for a different signal-to-noise ratio

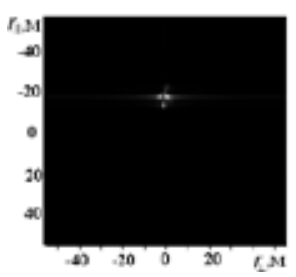

a)

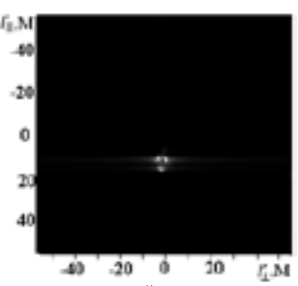

б)

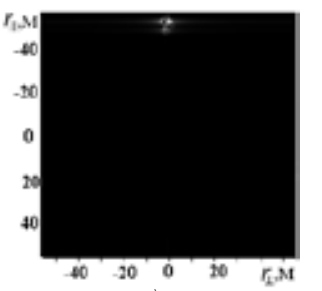

в)

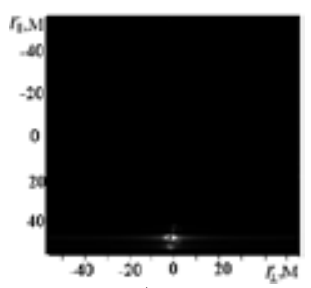

2)

Рис. 8. Двумерные радиолокационные изображения цели А-10А при различных ошибках измерения дальности

Fig. 8. Two-dimensional radar images of the target A-10A for various errors of range measurement

слева направо. Видно, что при отношении сигнал/шум - 2дБ распознавание становится проблематичным.

Производилась оценка влияния ошибок измерения дальности до цели на качество ДРЛИ. Моделировалось движение цели типа А-10А. Использовались предыдущие исходные данные за исключением: ракурс цели - 10; скорость полета цели - 200 м/с; ошибки измерения дальности \pm 20 и 70 м. На рис. 8 представлены ДРЛИ цели типа А-10А при отношении сигнал/шум 
13 дБ, ракурс q=10 и ошибках измерения дальности: $+20,-20$ м, +70 и -70 м соответственно слева направо, которые свидетельствуют о том, что среднеквадратические ошибки измерения дальности до цели приводят к смещению ДРЛИ по радиальной координате, что может быть скомпенсировано.

\section{Заключение}

Предложенная имитационная математическая модель построения двумерных радиолокационных изображений воздушных объектов позволяет проводить всесторонние исследования портретов 19 типов воздушных объектов зарубежного и российского производства при изменении ракурсов локации, характера и параметров траекторных нестабильностей полета, отношения сигнал/шум, параметров зондирующих сигналов РЛС с учетом ошибок измерения радиальной скорости и дальности до цели. Показано, что в условиях отсутствия дестабилизирующих факторов (неравномерности поворота, радиального перемещения, наличия шумов и помех и т.п.) формируемое двумерное радиолокационное изображение воздушных объектов адекватно отражает структуру цели.

Полученные результаты могут быть использованы при разработке и оценке качества систем распознавания классов и типов воздушных объектов по сигнальным признакам в существующих и перспективных РЛС.

\section{Список литературы}

[1] Стайнберг Б.Д. Формирование радиолокационного изображения самолета в диапазоне СВЧ. ТИИЭР. 1988, 76(12). 26-46 [Steinberg B.D. Formation of the radar plane in the microwave frequency range. TIIJeR, 1988, 76(12). 26-46 (in Russian)]

[2] Патент 2180445 (РФ) от 06.06.2000 г, МПК6 G 01 S 13/89. Способ построения двумерного радиолокационного изображения воздушной цели по траекторным нестабильностям ее полета / Митрофанов Д.Г., Гусев М.Д., Денисов А.В., Бортовик В.В. Заявка 2000114138/09. Приоритет 06.06.2000 г. [Patent 2180445 (RF), dated 06.06.2000, MPK6 G 01 S 13/89. A method of constructing a two-dimensional radiolocation image of air aim based on flight path instability. / Mitrofanov D.G., Gusev M.D, Denisov A.V., Bortovik V.V. Zaajvka 2000114138/09. Prioritet 06.06.2000 (in Russian)]

[3] Патент 2234110 (РФ), МПК7 G 01 S 13/89. Способ построения двумерного радиолокационного изображения воздушной цели / Бортовик В.В., Митрофанов Д.Г. и др. [Patent 2234110 (RF), MPK7 G 01 S 13/89. Method of constructing a two-dimensional radiolocation image of air aim. / Bortovik V.V., Mitrofanov D.G. et. al. (in Russian)]

[4] Ширман Я.Д., Горшков С.А., Лещенко С.П, Орленко В.М. Методы радиолокационного распознавания и их моделирование. Зарубежная радиоэлектроника: Успехи современной радиоэлектроники. Киев, 1996, 11, 3-62 [Shirman J.D., Gorshkov S.A., Leshchenko C.P., Orlenko V.M. Radar recognition Methods and modeling. Foreign Electronics: Achievements of modern electronics. Kiev, 1996, 11, 3-62 (in Russian)]

[5] Сухаревский О.И. Рассеяние электромагнитных волн воздушными и наземными радиолокаџионными объектами. Харьков, 2009 [Suhkarevskiy O.I. Scattering of electromagnetic waves by radar air and surface targets. Kharkov, 2009 (in Russian)]

$$
-773-
$$


[6] Миронов А.М., Митрофанов Д.Г., Помазуев О.Н., Бортовик В.В., Бердышев В.П., Цыганов А.А. Имитаиионная математическая модель построения и исследования радиолокационных портретов воздушных объектов в интересах распознавания. Свид. о регистр. электр. pecypca № 20498 от 19.11.2014 [Mironov A.M., Mitrophanov, D.G., Pomazuev O.N., Bortovik V.V., Berdishev V.P., Tsyganov A.A. Mathematical model building and Simulation study of radar portraits air objects for recognition. Certificate of registration of an electronic resource no. 20498, date of register. 19.11.2014 (in Russian)]

[7] Бердышев В.П., Миронов А.М., Помазуев О.Н., Стучилин А.И., Косолапов А.В. Программа, реализующая работу математической модели построения радиолокационных портретов воздушных объектов. Свид. о гос. регистр. прогр. для ЭВМ № 2015615457 от 18.05.2015 [Berdishev V.P., Mironov A.M, Pomazuev O.N., Stuchilin A.I., Kosolapov, A.V. Program that implements the work of mathematical model building radar portraits air targets. State registration Certificate of a computer programs no. 2015615457 date of registering. 18.05.2015 (in Russian)]

[8] Радиолокационные системы / В.П. Бердышев, Е.Н. Гарин, А.Н. Фомин и др. / под общ. ред. В.П. Бердышева. Красноярск, Сиб. федер. ун-т, 2011. 400 с. [Radiolokacionnie sistemi, V.P. Berdyshev, V.P. Berdyshev, E.N. Garin, A.N. Fomin e.al. / ed. by V.P. Berdyshev. Krasnoajrsk, Sib. Feder. Un-t, 2011, 400 p. (in Russian)]

[9] Основы построения РЛС РТВ / В.Н. Тяпкин, В.П. Бердышев, Е.Н. Гарин и др. / под общ. ред. В.Н. Тяпкина. Красноярск, Сиб. федер. ун-т, 2011. 536 с. [Basics of creation RLS RTT. / V.N. Tajpkin, V.P. Berdyshev, E.N. Garin et. al. / ed. by V.N. Tajpkin. Krasnoajrsk, Sib. Feder. Un-t, 2011, 536 p. (in Russian)] 